\title{
Minimally invasive percutaneous transpedicular screw fixation: increased accuracy and reduced radiation exposure by means of a novel electromagnetic navigation system
}

\author{
Ron von Jako • Michael A. Finn • \\ Kenneth S. Yonemura • Ali Araghi • Larry T. Khoo • \\ John A. Carrino • Mick Perez-Cruet
}

Received: 21 July 2010 /Accepted: 12 November 2010 /Published online: 14 December 2010

(C) The Author(s) 2010. This article is published with open access at Springerlink.com

\begin{abstract}
Background Minimally invasive percutaneous pedicle screw instrumentation methods may increase the need for intraoperative fluoroscopy, resulting in excessive radiation exposure for the patient, surgeon, and support staff. Electromagnetic field (EMF)-based navigation may aid more accurate placement of percutaneous pedicle screws while reducing fluoroscopic exposure. We compared the accuracy, time of insertion, and radiation exposure of EMF with traditional fluoroscopic percutaneous pedicle screw placement.
\end{abstract}

\section{R. von Jako}

GE Healthcare,

Boston, MA, USA

M. A. Finn $\cdot$ K. S. Yonemura

Department of Neurosurgery, University of Utah,

Salt Lake City, UT, USA

A. Araghi

Texas Back Institute,

Scottsdale, AZ, USA

L. T. Khoo

University of California,

Los Angeles, CA, USA

J. A. Carrino

Department of Radiology, Johns Hopkins University,

Baltimore, MD, USA

M. Perez-Cruet

Department of Neurosurgery, William Beaumont Hospital,

Royal Oak, MI, USA

R. von Jako $(\bowtie)$

GE Healthcare,

301 Ballardvale St,

Wilmington, MA 01887, USA

e-mail: drvonjako@comcast.net
Methods Minimally invasive pedicle screw placement in T8 to S1 pedicles of eight fresh-frozen human cadaveric torsos was guided with EMF or standard fluoroscopy. Setup, insertion, and fluoroscopic times and radiation exposure and accuracy (measured with post-procedural computed tomography) were analyzed in each group.

Results Sixty-two pedicle screws were placed under fluoroscopic guidance and 60 under EMF guidance. Ideal trajectories were achieved more frequently with EMF over all segments $(62.7 \%$ vs. $40 \% ; p=0.01)$. Greatest EMF accuracy was achieved in the lumbar spine, with significant improvements in both ideal trajectory and reduction of pedicle breaches over fluoroscopically guided placement ( $64.9 \%$ vs. $40 \%, p=0.03$, and $16.2 \%$ vs. $42.5 \%, p=0.01$, respectively). Fluoroscopy time was reduced $77 \%$ with the use of EMF ( $22 \mathrm{~s}$ vs. $5 \mathrm{~s}$ per level; $p<0.0001$ ) over all spinal segments. Radiation exposure at the hand and body was reduced $60 \%(p=0.058)$ and $32 \%(p=0.073)$, respectively. Time for insertion did not vary between the two techniques. Conclusions Minimally invasive pedicle screw placement with the aid of EMF image guidance reduces fluoroscopy time and increases placement accuracy when compared with traditional fluoroscopic guidance while adding no additional time to the procedure.

Keywords Minimally invasive - Electromagnetic field navigation $\cdot$ Pedicle screw . Fluoroscopy $\cdot$ Accuracy

\section{Introduction}

Pedicle screw fixation provides superior biomechanical strength when compared with wiring or hook-based constructs for creation of a stable environment for spinal fusion 
$[5,12,22,31,36]$. Standard methods of pedicle screw instrumentation require significant exposure of the posterior bony elements of the spine to provide landmarks from which to guide the placement of instrumentation. Such exposure is associated with significant amounts of blood loss [17], as well as paraspinal muscular injury, which has been associated with postoperative back pain $[18,19]$. The accuracy of placement of pedicle screws has been shown to be improved with the use of frameless stereotaxic image guidance in most [2, 3, 21, 23] but not all [13] studies. Adoption of image-guided surgical techniques has been limited to date amid concerns of increased operative time and complexity of the technology [2]. Recently, developments in percutaneous pedicle screw fixation offer a less traumatic approach resulting in less damage to the surrounding musculature, decreased blood loss, and decreased recovery time $[15,16,20]$. These characteristics may eventually translate into improved long-term results, with decreased muscle denervation, atrophy, and pain [30]. Accurate minimally invasive pedicle screw placement is complicated, however, by the obscuration of normal anatomical landmarks. Errors in placement are therefore a primary concern, with one study reporting almost $10 \%$ of patients needing revision surgery [30]. Additionally, the technique depends heavily on fluoroscopic guidance, which can result in significant levels of radiation exposure to both the surgeon and assistant [28]. An efficient image-guided system that allows accurate pedicle screw placement and a reduction in the amount of fluoroscopic time would therefore be of value.

Computer-aided fluoroscopic techniques [6, 9, 27, 29, 34] are among the various methodologies devised to increase the accuracy of screw placement; however, widespread adoption has been slow because of the increased set-up and operative times and lack of perceived benefit [3]. Electromagnetic field (EMF) navigation-based systems eliminate cumbersome optical array receivers as well as line-of-sight issues that can interfere with the normal flow of the operative procedure $[10,25]$. The tradeoff for this flexibility is the limited size of the EMF field (18-in. radial from center of EMF transmitter; Fig. 1). In addition, large ferromagnetic instruments can create distortion within the EMF field. Studies have confirmed that the accuracy of pedicle screw placement is similar using either optical or EMF-based navigation systems [6, 9, 27, 29, 35].

Although the results of stereotaxic guidance for pedicle screw placement in open procedures have been promising, a relative paucity of laboratory and clinical data exist regarding its use in a minimally invasive approach $[1,7,8,14]$. To date, no laboratory work has been done to compare the radiation exposure to the user using dosimeter measurements. This study aims to compare the accuracy, time of insertion, and radiation from EMF-guided placement of minimally invasive

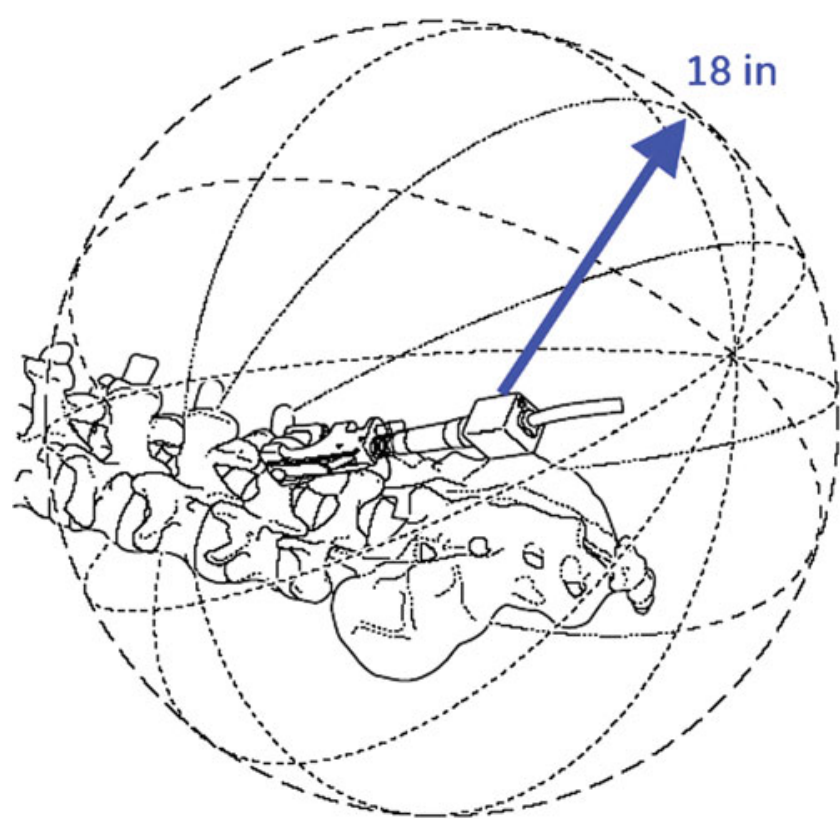

Fig. 1 Drawing showing that an 18-in. field is one of the limiting factors in the use of electromagnetic guidance

pedicle screws with the traditional fluoroscopically guided approach.

\section{Materials and methods}

The spines of eight fresh-frozen human cadavers with intact spines from at least $\mathrm{T} 8$ to the sacrum were prescreened for osseous vertebral pathological conditions, including evidence of tumor infiltration, traumatic disruption, or past surgical intervention. Intact cadaver torsos were placed in the prone position on radiolucent tables.

A GE OEC 9900 Elite fluoroscope with an integrated navigation system (GE Healthcare, Salt Lake City, UT; not commercially available) was used for fluoroscopic imaging (Fig. 2). To eliminate bias and confirm that the navigation system works with multiple screw constructs, two minimally invasive percutaneous pedicle screw systems that had been modified for use in an EMF environment were used: the Paramount (Integra Spine, Burlington, MA, USA) and the Pathfinder (Zimmer Spine, Austin, TX, USA) systems. Titanium screw extenders for each system were created to avoid interference within the EMF field that would be expected from the normal stainless steel extenders. A special bone biopsy needle known as the Nav Access Needle [33], which provides stable attachment of an EMF receiver, was used for Kirschner wire (K-wire) placement. A bone pin or spinous process clamp was used to attach the EMF dynamic reference transmitter to the spinous process 


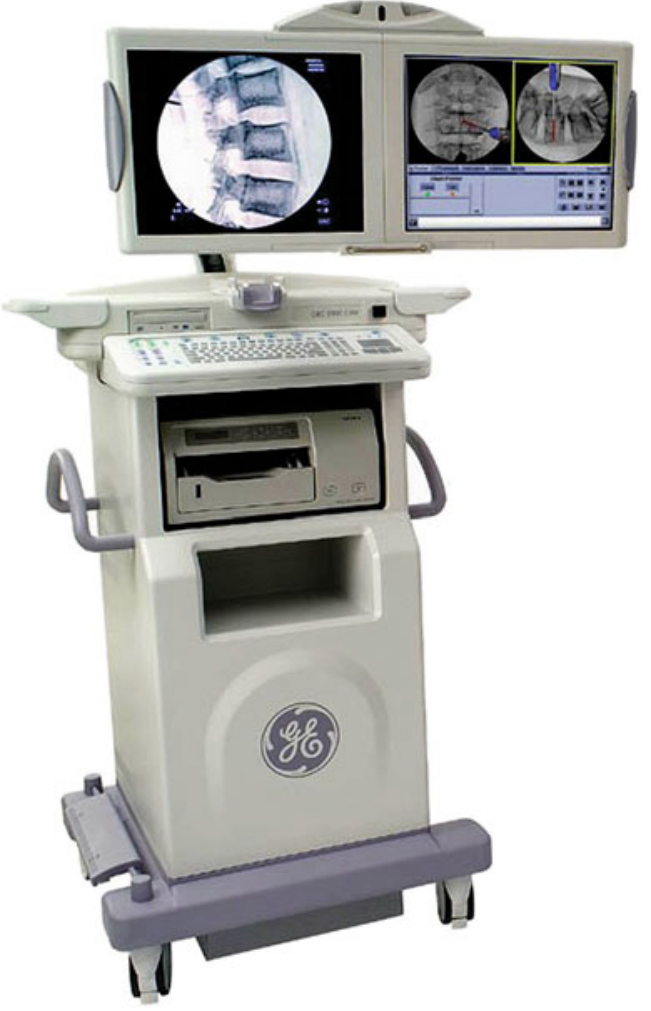

Fig. 2 Photograph showing the GE OEC 9900 Elite fluoroscopic display

of the level adjacent to the targeted vertebrae as previously described (Fig. 3) [11, 35]. The typical montage for transmitter placement was on the spinous processes of T11, L2, and L5, as the system allows for accurate navigation of up to three vertebrae from each transmitter location. For two cadavers, T8 was also used for placement of the EMF transmitter.

Four attending spinal surgeons (AA, LTK, MPC, and $\mathrm{KSY}$ ) with experience in minimally invasive spine surgery

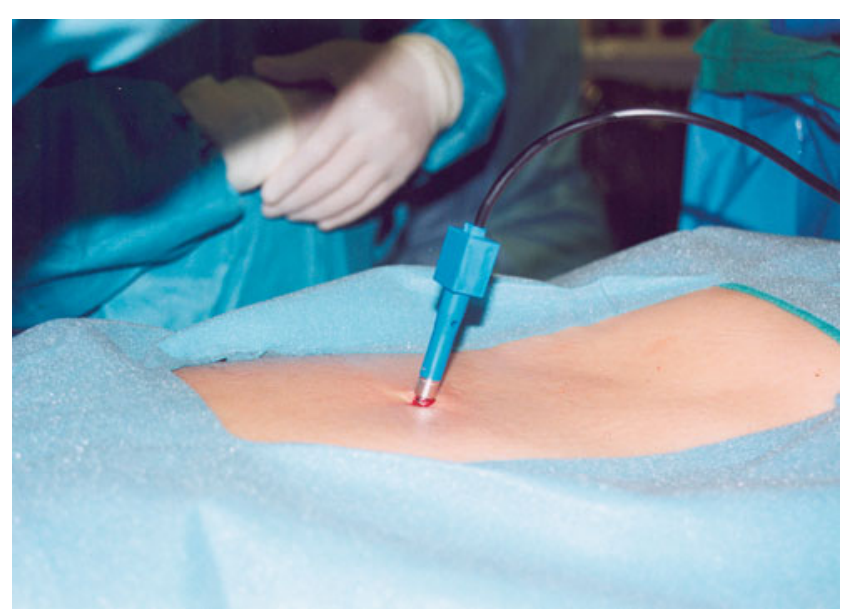

Fig. 3 Photograph showing the bone pin inserted into spinous process and percutaneous pedicle screw instrumentation worked in pairs on each cadaver.

Procedure

Each cadaver was assigned to implantation with either the Pathfinder or the Paramount percutaneous pedicle screw system. Screw placement with EMF navigation (Nav) and standard fluoroscopic imaging (Fluoro) was compared between alternating segments rather than alternating technique from side to side. A comparison by level, in which one technique is performed bilaterally, was thought to reduce a potential bias for a reduction in time and improved accuracy based on knowledge gained from placement of the first screw when placing the contralateral screw. A comparison by level is also more consistent with the manner in which surgery is performed with sequential placement of screws bilaterally before moving to the next level.

The S1 level was allocated to either the Nav or Fluoro group on an alternate basis to ensure the numbers for this level were evenly randomized. All other levels starting at L5 and moving upward were randomly allocated based on a coin flip.

After placement of the EMF transmitter, anteroposterior (AP) and lateral fluoroscopic views of the vertebrae were saved on the Nav system. Transpedicular K-wires were then placed percutaneously using either the image guidance Nav Access needle or a standard Jamshidi needle with the aid of live AP and lateral fluoroscopic imaging (Figs. 4 and 5).

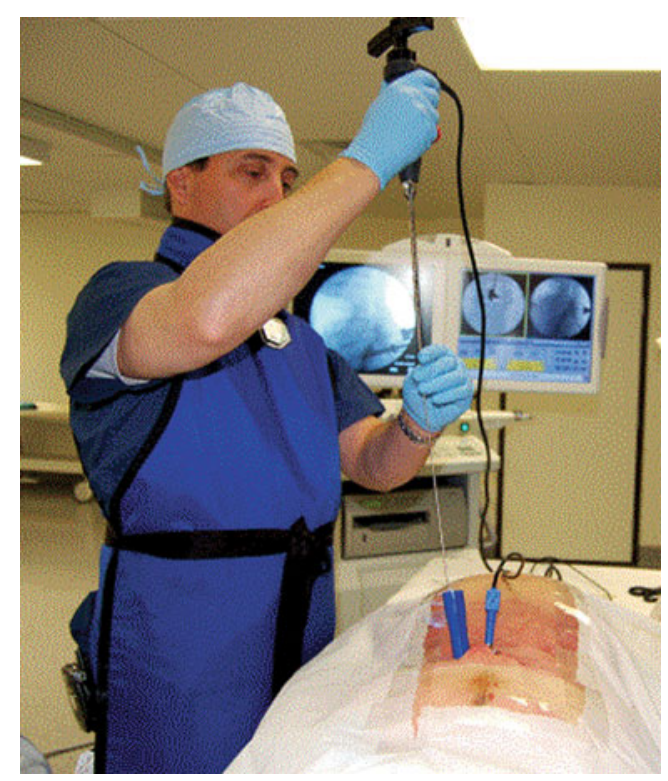

Fig. 4 Photograph showing how the K-wires are inserted through the Nav access needle with EMF receiver 

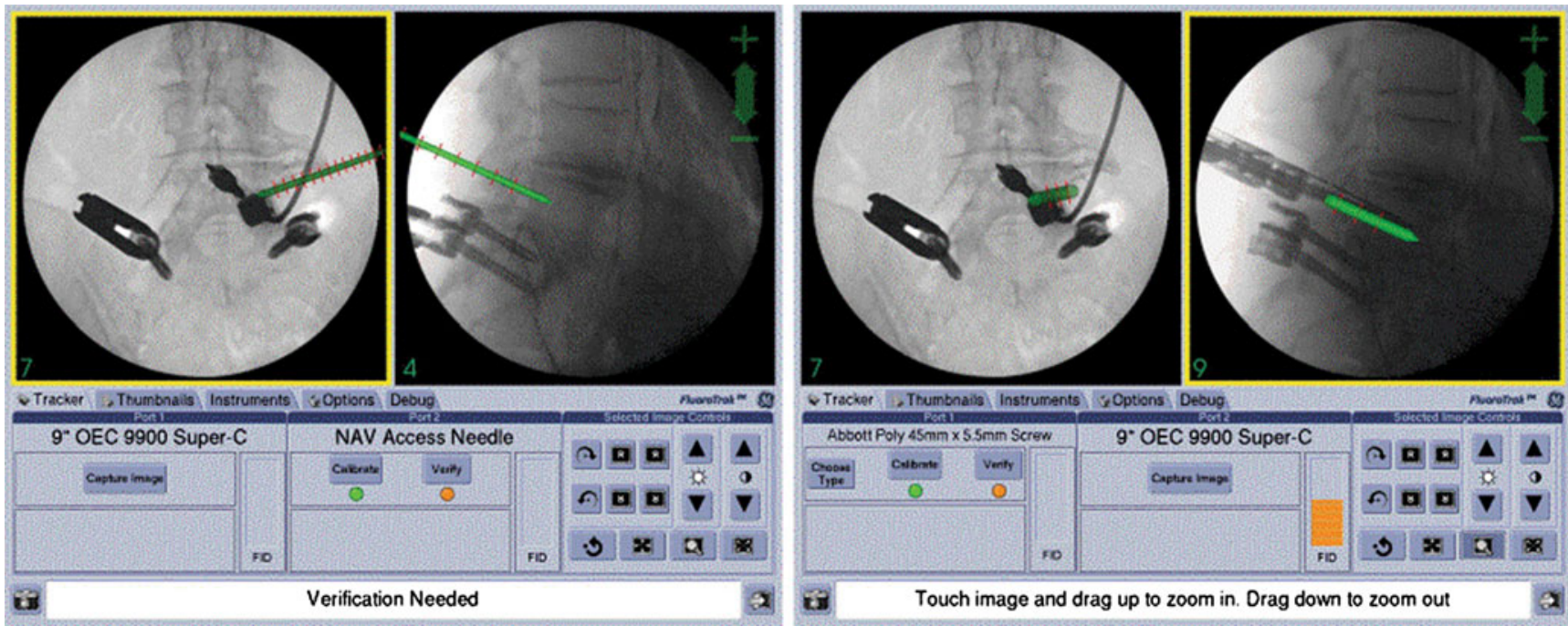

Fig. 5 a NAV screen demonstrating target of the right pedicle at L5. b Lateral fluoroscopic capture showing the overlay of the planning trajectory and actual screw position

The percutaneous pedicle screw systems used similar tools, which required placement over the transpedicular K-wires. In the Nav group, these were all image guided and placed using the EMF cannulated T-handle, which allowed for real-time biplanar visualization of the trajectory and progression of the instrument through the pedicle. Standard fluoroscopic technique was used in the Fluoro group. The screw extenders and $\mathrm{K}$-wires were removed, leaving the screws in place for subsequent computed tomography (CT) scan evaluation of screw placement accuracy.

\section{Outcome measures}

Intraoperative measured variables included set-up, insertion, and fluoroscopy times. Insertion time measurements included set-up and initial image capture time for both Nav and Fluoro groups. Radiation exposure was detected by hand and ring thermoluminescent dosimeter badges. Each screw was assessed for pedicle, vertebral, and critical breach on post-procedural $\mathrm{CT}$ reconstruction scans. The ideal screw trajectory, defined as convergence of the pedicle screws at the ventral aspect of the vertebral body and totally within the pedicle, was determined. A 4-point grading scale was used as follows: grade 0 (ideal) - accurate screw with no perforation through any cortex; grade 1 (minimally displaced) - safe screw with cortical perforation of $<3 \mathrm{~mm}$; grade 2 (moderately displaced) — displaced by $\geq 3 \mathrm{~mm}$ but $\leq$ $5 \mathrm{~mm}$; and grade 3 (critical perforation)—displaced by $>5 \mathrm{~mm}$.

The accuracy of each technique over each spinal zone (thoracic, lumbar, and sacral) was statistically analyzed using a chi-square test with $p=0.05$ considered significant.

\section{Results}

In total, 122 pedicle screws were placed, 62 under fluoroscopic guidance and 60 under EMF guidance (Table 1). Seventy-eight of these were in the lumbar pedicle, 28 were in the thoracic spine, and 16 were in the sacral spine.

Accuracy of placement over all segments, as assessed by pedicle and vertebral body breaches, was slightly better in the EMF Nav group than in the conventional fluoroscopic C-arm group, but this difference was not statistically significant: Pedicle breaches were seen in $17 \%$ of EMFplaced screws and $29 \%$ of fluoroscopically placed screws ( $p=0.12$ ), while vertebral body breaches were seen in $1.7 \%$ of the EMF-placed screws and $4.8 \%$ of the fluoroscopically placed screws $(p=0.33)$ (Table 1). Accuracy of placement of lumbar pedicle screws was significantly improved with the use of EMF (16.2\% pedicle breach vs. $42.5 \%$ with fluoroscopic; $p=0.01$ ), but there was no significant decrease in cortical breaches with the use of EMF in the thoracic or sacral regions.

Ideal screw trajectories were achieved more often with EMF guidance over all spinal segments $(62.7 \%$ vs. $40 \%$, $p=0.01)$. This effect was most pronounced in the lumbar segments, where $64.9 \%$ of screws placed with EMF guidance achieved ideal trajectory vs. $40 \%$ placed with fluoroscopy $(p=0.03)$. No grade 3 trajectories occurred with standard fluoroscopy or EMF navigation, and most trajectories in either group were grade 0 or 1 (Table 1). There was no significant difference between the two techniques over other segments.

Insertion times, including set-up time, between the two techniques did not significantly differ overall for the two 
Table 1 Summary of pedicle screw placement data comparing electromagnetic navigation and fluoroscopy

\begin{tabular}{llll}
\hline & EM group & Fluoroscopy group & $p$ value \\
\hline Number of pedicle screws placed & 60 & 62 & \\
Total insertion and setup time (s) & 923 & 952 & 0.6911 \\
Average fluoroscopy time per level (s) & 5 & 22 & $<0.0001^{*}$ \\
Number of pedicle breaches (\%) & $10(17)$ & $18(29)$ & 0.12 \\
Number of lumbar pedicle breaches (\%) & $6(16.2)$ & $17(42.5)$ & 0.01 \\
Number of vertebral body breaches (\%) & $1(1.7)$ & $3(4.8)$ & 0.33 \\
Number of ideal (grade 0) trajectories (\%) & $37(62.7)$ & $25(40)$ & $0.01^{*}$ \\
Number of grade 1 trajectories (\%) & $18(30.5)$ & $35(56)$ & \\
Number of grade 2 trajectories (\%) & $4(6.8)$ & $2(3.2)$ & 0 \\
Number of grade 3 trajectories (\%) & 0 & $16(40)$ & $0.03 *$ \\
Ideal (grade 0) lumbar trajectories (\%) & $24(64.9)$ & 20.2 & 0.073 \\
Radiation exposure in body (mrem) & 13.8 & 37.5 & 0.058 \\
Radiation exposure in hand (mrem) & 15.0 & & \\
\hline
\end{tabular}

groups (923 s with EMF vs. $952 \mathrm{~s}$ with Fluoro, $p=0.6911$ ), and this was also true for any spinal segment analyzed separately. Radiation time, however, was significantly reduced over all segments (5 s per level with EMF vs. $22 \mathrm{~s}$ per level with Fluoro, $p<0.0001)$. Highly significant reductions in radiation time using EMF vs. Fluoro guidance were seen over all spinal segments upon individual blinded analysis by the radiologist (JAC).

Total body and hand radiation doses seen by the operating surgeons were decreased with the use of EMF, although these results were just less than significant (13.8 vs. $20.2 \mathrm{mrem}, p=0.073$, and 15.0 vs. $37.5 \mathrm{mrem}, p=0.058$, for body and hand, respectively). Of note, all surgeons attempted to reduce radiation exposure by stepping away from the operative table during image acquisition for both the Nav and Fluoro groups, which is reflective of their experience using fluoroscopy.

\section{Discussion}

Although the literature has not confirmed that image guidance is superior to fluoroscopic guidance in traditional open procedures for placing pedicle screws, few studies have compared these techniques for placement of percutaneous pedicle screws. Fewer studies have analyzed radiation exposure using the two techniques, and none, to our knowledge, have analyzed recent EMF technology. Accuracy of pedicle screw placement in this study was measured by assessment of cortical breach in both the pedicle and vertebral body as well as by subjective assessment of ideal trajectory. We found that accuracy of placement of lumbar pedicle screws was significantly improved by both of these measures; however, the accuracy was not significantly improved for thoracic and sacral screws. Significant gains in pedicle screw placement accuracy with guidance in the lumbar spine have also been demonstrated in a recent, large meta-analysis [21].

Cortical breach has been the most commonly used measure in determining pedicle screw accuracy [21], but there is significant variability in the definition of these findings in the literature, making comparisons between series difficult. Kosmopoulous and Schizas [21] reported on the use of 35 different methods of assessment in their metaanalysis and noted that approximately $50 \%$ of studies making claims about the accuracy of placement did not clearly define how accuracy was assessed. Even when cortical breach is used as a parameter, considerable variation exists between studies. Some authors have reported only a cortical breach of $>2 \mathrm{~mm}$ and others reported only on the direction of breach [21, 30]. The assessment of cortical breach is straightforward on postprocedural CT scans and should represent a minimum reporting requirement to serve as a basis for comparison between studies. The amount and direction of breach are secondary measures that may or may not have significance in relation to the potential of neural injury [32]; that is, medial cortical breaches have more significance than lateral pedicle cortex breaches.

Although the subgroup analysis indicated that accuracy with navigation was improved in the lumbar spine, increased accuracy of pedicle screw placement in the thoracic spine was not illustrated in this study. In fact, the results suggested a trend toward greater inaccuracy with the EMF navigation system. Similar results have been reported in another study [13], but this is not a universal finding [2, 3]. Kosmopoulos and Schizas [21] noted similar findings in the thoracic group in their metaanalysis. They reported a decreased median accuracy of thoracic pedicle screw placement in vivo with the use of 
navigation compared with fluoroscopy alone (94.3\% accurate vs. $82.2 \%$ ) and very similar results in cadaver studies (74.9\% accurate with navigation and $74.5 \%$ without). Possible explanations for this finding are that the learning curve needed to master the navigation technology is steep, and the decreased diameter of the thoracic pedicle makes screw placement inaccuracies more prevalent.

The accuracy of screw placement is of great importance with placement of percutaneous pedicle screws, but operative and radiation exposure times must also be considered. Time for insertion with the EMF technique (and thus operative time) did not vary from that of the standard fluoroscopically guided technique in the current study, which is especially significant because the version of the navigation system in this study was new to all participating surgeons. Thus, shorter operative times would be expected with further familiarity with the navigation system and instrument refinement. Although increased speed has not been shown in other studies, this can be a reasonably expected byproduct of navigation because manipulation of a fluoroscope during surgery can be time consuming, especially in minimally invasive surgeries that require AP and lateral imaging for each level. Elimination of the required movement of the fluoroscope around the operative field should also reduce the potential for contamination of the operative field and concern for infection [4]. Additionally, simultaneous viewing of both AP and lateral projections facilitates percutaneous screw placement.

An additional outcome measure in this study was total fluoroscopic time and total radiation exposure. The reduction in fluoroscopic time with EMF was highly significant, but the reduction in radiation exposure measured on the dosimeters was not statistically significant. This is likely due to each surgeon's habit of backing away from the fluoroscope with each exposure, which may provide some protection to the surgeon. Although it was not measured with dosimeters, the highly significant reduction in fluoroscopic time should reduce the exposure to the patient. Total fluoroscopic time and radiation exposure are directly related, and any reduction in exposure time should be of benefit for the patient and the operating room staff. This finding holds special implications for the spine surgeon, as radiation exposure in spinal surgery has been demonstrated to be 10-12 times that of other musculoskeletal surgeries and has the potential to exceed recommended yearly allowances [28]. Factors unique to spine surgery that may contribute to increased exposure include the increased penetrating beam energy requirements to image the spine adequately [24], proximity of the surgeon's hands to the field (which may be exacerbated by the need to maintain alignment of instrumentation), increased Compton scatter at the beam entry site, and the frequent necessity of having either the surgeon or assistant standing next to the beam generator [28]. Furthermore, poor technique in which the hands are directly irradiated can dramatically increase exposure to as high as $4,000 \mathrm{mrem} / \mathrm{min}$ (recommended yearly hand allowance is $50,000 \mathrm{mrem}$ ) [26]. While the consequences of chronic radiation exposure are, as yet, unknown, the increasing exposure beyond recommended limits is certainly cause for concern, especially as minimally invasive techniques requiring fluoroscopy increase in frequency.

Potential disadvantages of the EMF navigation system over traditional fluoroscopy could include system set-up and registration time and the chance of software failure. In addition, there is the need for additional incisions and time to place the spinous process tracking system and the possibility of displacement of the spinous process tracking system. Redo operations in patients in whom the spinous process has been removed previously would present a challenge. Inaccuracies could also be associated with shifting of the attached dynamic reference transmitter position during applications of pedicle screws. Resecuring the bumped transmitter with updated fluoroscopic images allows continued navigation. Minor inaccuracies (i.e., $<3 \mathrm{~mm}$ ) might be of less importance in targeting the L4 to S1 pedicles, as these are the more frequently instrumented pedicles. The 18-in. surgical field may present a potential limitation in the use of EMF navigation when tracking some elongated instruments used in percutaneous approaches.

\section{Conclusions}

The use of EMF navigation for the insertion of percutaneous pedicle screw instrumentation was found to increase accuracy in the lumbar spine. The increase in accuracy is similar to those reported in the literature for optical-based navigation techniques. A significant reduction in fluoroscopic time was also noted in the EMF navigation group. This is an important factor in minimally invasive surgery, which requires a more extensive use of fluoroscopy. The use of EMF navigation avoids the cumbersome line-of-sight issues of the opticalbased systems; thus, it may facilitate minimally invasive percutaneous procedures. Guidance should, therefore, be considered a valuable tool in the spine surgeon's armamentarium, especially when applying instrumention in revision or deformity spine surgery.

Acknowledgments Zimmer Spine and Integra Life Sciences provided significant support in prototypes for this study. We thank Cindy Ochampaugh of Landauer, Inc. (Glenwood, IL) for providing us with the numerous versions and samples of dosimeters and the accurate readings of them at no cost. We thank Kristin Kraus, M.Sc., for her editorial assistance in preparing this paper. We also recognize the late Michael Hurd for his support. 


\section{Conflicts of interest None.}

Financial support Zimmer Spine and Integra Life Sciences provided significant support in prototypes for this study.

Disclosure Ron Von Jako is an employee of GE Healthcare. Larry T. Khoo serves as a consultant for Zimmer Spine, GE, and Integra Life Sciences. Ali Araghi is a consultant, stockholder, and design surgeon with Integra Life Sciences, a consultant for Zimmer Spine, and an Advisory Board member for GE. Ken Yonemura has consulting agreements with Zimmer Spine and GE. Mick Perez-Cruet has consulting agreements with Zimmer Spine and GE.

Open Access This article is distributed under the terms of the Creative Commons Attribution Noncommercial License which permits any noncommercial use, distribution, and reproduction in any medium, provided the original author(s) and source are credited.

\section{References}

1. Acosta FL Jr, Thompson TL, Campbell S, Weinstein PR, Ames CP (2005) Use of intraoperative isocentric C-arm 3D fluoroscopy for sextant percutaneous pedicle screw placement: case report and review of the literature. Spine J 5:339-343

2. Assaker R, Reyns N, Vinchon M, Demondion X, Louis E (2001) Transpedicular screw placement: image-guided versus lateral-view fluoroscopy: in vitro simulation. Spine 26:2160-2164

3. Austin MS, Vaccaro AR, Brislin B, Nachwalter R, Hilibrand AS, Albert TJ (2002) Image-guided spine surgery: a cadaver study comparing conventional open laminoforaminotomy and two image-guided techniques for pedicle screw placement in posterolateral fusion and nonfusion models. Spine 27:25032508

4. Biswas D, Bible JE, Whang PG, Simpson AK, Grauer JN (2008) Sterility of C-arm fluoroscopy during spinal surgery. Spine 33:1913-1917

5. Brodke DS, Dick JC, Kunz DN, McCabe R, Zdeblick TA (1997) Posterior lumbar interbody fusion. A biomechanical comparison, including a new threaded cage. Spine 22:26-31

6. Choi WW, Green BA, Levi AD (2000) Computer-assisted fluoroscopic targeting system for pedicle screw insertion. Neurosurgery 47:872-878

7. Foley KT, Gupta SK (2002) Percutaneous pedicle screw fixation of the lumbar spine: preliminary clinical results. J Neurosurg 97:7-12

8. Foley KT, Holly LT, Schwender JD (2003) Minimally invasive lumbar fusion. Spine 28:S26-S35

9. Foley KT, Simon DA, Rampersaud YR (2001) Virtual fluoroscopy: computer-assisted fluoroscopic navigation. Spine 26:347351

10. Foley KT, Smith MM (1996) Image-guided spine surgery. Neurosurg Clin N Am 7:171-186

11. Fraser J, Von Jako R, Carrino J, Härtl R (2008) Electromagnetic navigation in minimally invasive spine surgery: results of a cadaveric study to evaluate percutaneous pedicle screw insertion. SAS J 2:43-47

12. Gaines RW Jr (2000) The use of pedicle-screw internal fixation for the operative treatment of spinal disorders. J Bone Joint Surg Am 82-A:1458-1476
13. Hart RA, Hansen BL, Shea M, Hsu F, Anderson GJ (2005) Pedicle screw placement in the thoracic spine: a comparison of image-guided and manual techniques in cadavers. Spine 30:E326E331

14. Holly LT, Foley KT (2003) Three-dimensional fluoroscopyguided percutaneous thoracolumbar pedicle screw placement. Technical note. J Neurosurg 99:324-329

15. Isaacs RE, Podichetty VK, Santiago P, Sandhu FA, Spears J, Kelly K, Rice L, Fessler RG (2005) Minimally invasive microendoscopyassisted transforaminal lumbar interbody fusion with instrumentation. J Neurosurg Spine 3:98-105

16. Jang JS, Lee SH (2005) Minimally invasive transforaminal lumbar interbody fusion with ipsilateral pedicle screw and contralateral facet screw fixation. J Neurosurg Spine 3:218-223

17. Johnson RG, Murphy M, Miller M (1989) Fusions and transfusions. An analysis of blood loss and autologous replacement during lumbar fusions. Spine 14:358-362

18. Kawaguchi Y, Matsui H, Tsuji H (1994) Back muscle injury after posterior lumbar spine surgery. Part 2: histologic and histochemical analyses in humans. Spine 19:2598-2602

19. Kawaguchi Y, Matsui H, Tsuji H (1996) Back muscle injury after posterior lumbar spine surgery. A histologic and enzymatic analysis. Spine 21:941-944

20. Kim DY, Lee SH, Chung SK, Lee HY (2005) Comparison of multifidus muscle atrophy and trunk extension muscle strength: percutaneous versus open pedicle screw fixation. Spine 30:123129

21. Kosmopoulos V, Schizas C (2007) Pedicle screw placement accuracy: a meta-analysis. Spine 32:E111-E120

22. Krag MH, Beynnon BD, Pope MH, Frymoyer JW, Haugh LD, Weaver DL (1986) An internal fixator for posterior application to short segments of the thoracic, lumbar, or lumbosacral spine. Design and testing. Clin Orthop Relat Res 203:75-98

23. Laine T, Schlenzka D, Makitalo K, Tallroth K, Nolte LP, Visarius H (1997) Improved accuracy of pedicle screw insertion with computer-assisted surgery. A prospective clinical trial of 30 patients. Spine 22:1254-1258

24. Little J, Taveras J (1992) Radiology: diagnosis, imaging intervention. JB Lippincott, Philadelphia

25. Maciunas R (1997) Interactive image guided neurosurgery. In: Tindall G, Cooper P, Barrow D (eds) The practice of neurosurgery. Williams \& Wilkins, Philadelphia

26. Mehlman CT, DiPasquale TG (1997) Radiation exposure to the orthopaedic surgical team during fluoroscopy: "how far away is far enough?" J Orthop Trauma 11:392-398

27. Quinones-Hinojosa A, Robert Kolen E, Jun P, Rosenberg WS, Weinstein PR (2006) Accuracy over space and time of computerassisted fluoroscopic navigation in the lumbar spine in vivo. $\mathrm{J}$ Spinal Disord Tech 19:109-113

28. Rampersaud YR, Foley KT, Shen AC, Williams S, Solomito M (2000) Radiation exposure to the spine surgeon during fluoroscopically assisted pedicle screw insertion. Spine 25:2637-2645

29. Resnick DK (2003) Prospective comparison of virtual fluoroscopy to fluoroscopy and plain radiographs for placement of lumbar pedicle screws. J Spinal Disord Tech 16:254-260

30. Ringel F, Stoffel M, Stuer C, Meyer B (2006) Minimally invasive transmuscular pedicle screw fixation of the thoracic and lumbar spine. Neurosurgery 59:ONS361-ONS367

31. Sasso RC, Cotler HB (1993) Posterior instrumentation and fusion for unstable fractures and fracture-dislocations of the thoracic and lumbar spine. A comparative study of three fixation devices in 70 patients. Spine 18:450-460

32. Schulze CJ, Munzinger E, Weber U (1998) Clinical relevance of accuracy of pedicle screw placement. A computed tomographicsupported analysis. Spine 23:2215-2221 
33. von Jako $R$ (2006) Lumbosacral spine navigation and visualization of an access needle system. US patent no. 20060063998

34. von Jako R, Cselik Z (2009) Percutaneous laser discectomy with stereotactic computer-assisted surgical navigation. J Lasers Surg Med 41:42-51

35. von Jako RA, Carrino JA, Yonemura KS, Noda GA, Zhue W, Blaskiewicz D, Rajue M, Groszmann DE, Weber G (2009) Electromagnetic navigation for percutaneous guidewire insertion: accuracy and efficiency compared to conventional fluoroscopic guidance. Neuroimage 47(Suppl 2):T127T132

36. Zindrick MR, Wiltse LL, Widell EH, Thomas JC, Holland WR, Field BT, Spencer CW (1986) A biomechanical study of intrapeduncular screw fixation in the lumbosacral spine. Clin Orthop Relat Res 203:99-112

\section{Comment}

This article reports on electromagnetically controlled minimally invasive pedicle screw insertion in the TL spine including $\mathrm{S} 1$, on cadavers, and reports greater accuracy and less radiation exposure than with more "traditional" fluoroscopy-based screw insertion. This seems a promising step toward facilitating screw insertion with desired radiation reduction. The article points in the right direction, but does not resolve the ongoing controversy between advantages and disadvantages of "minimally" invasive vs. "open." Furthermore, electromagnetical guidance may facilitate both open and "minimally invasive" techniques.

Michael Payer

Geneva, Switzerland 